\title{
SPATIAL RESOLUTION IN GNSS-R UNDER COHERENT SCATTERING
}

\author{
A. Camps \\ CommSensLab Unidad María de Maeztu, Dept. of Signal Theory and Communications, \\ Universitat Politècnica de Catalunya - BarcelonaTech Remote Sensing Lab. and IEEC/CTE-UPC, \\ UPC Campus Nord, D4-016; 08034 Barcelona, Spain Tel. +34 93 4054153, Fax +34 93 4017232, \\ E-mail: camps@tsc.upc.edu
}

\begin{abstract}
GNSS-R can be understood as a multi-static radar using satellite navigation signals as signals of opportunity. The scattered signals over sea ice, flooded areas -even under dense vegetation-, and in some cases over land show a significant coherent component. Under coherent scattering conditions, it is usually stated that the coherent signal component comes from an area equal to the first Fresnel zone. This work analyzes in more detail the spatial resolution in this forward scattering configuration, showing that, when coherent scattering is non-negligible, the spatial resolution is mostly determined by the geometry, and not by typical surface roughness values. As the scattering area around the specular reflection point increases and encompasses the first Fresnel zone, the received power increases and then it fluctuates as higher order Fresnel zones are included (rapid phase changes due to the spherical waves). These contributions may explain in part the large scattering encountered over inhomogeneous land regions, as these different contributions add or subtract, depending on the phase of the electric field, and are weighted by different scattering coefficients (i.e. changes in the dielectric constant and/or surface roughness, such in water ponds, or some agricultural fields...). Finally, over homogeneous targets, when all Fresnel zones are included, the received power tends asymptotically to the value obtained using the free-space propagation with a total path length equal to the sum of the path lengths, weighted by the reflection coefficient. This value can also be interpreted as coming from an effective region that is actually $\sim 0.6$ times the first Fresnel zone.
\end{abstract}

Index Terms - GNSS-R, coherent scattering, spatial resolution, Fresnel zone

\section{INTRODUCTION}

In GNSS-R, the presence of a significant coherent component in the scattered signals has been identified in space-borne data from the UK TDS-1 GNSS-R experiment notably over sea ice $[1,2,3]$, over land from the BEXUS 17 and 19 stratospheric balloon flights (e.g. see I/Q plots of the demodulated reflected signal in fig. 12 of [4], or fig. 3 of [5]) using dual-frequency observations and wide-band (P) codes over land as well, over calm ocean using UK TDS-1 data [6], or even from rough ocean from a cliff (e.g see fig. 3 of [7] where the phase of the peak of the delayDoppler map was tracked at $12 \mathrm{~m} / \mathrm{s}$ wind speed), and more recently over flooded areas using CYGNSS data [8]. In [9], Martin et al. proposed a technique to estimate the ratio of the coherent to incoherent reflected powers which was estimated to be over the ocean as high as $0.08 \%$ from space (TDS-1), $0.4 \%$ from a plane at $3 \mathrm{~km}$ height, and $3 \%$ from a
$121 \mathrm{~m}$ cliff even under high wind conditions. These values indicate that high sensitive GNSS receivers could even potentially track the coherent component as typical thresholds for code phase and carrier phase tracking are $\sim 14 \mathrm{~dB}$ and $\sim 7 \mathrm{~dB}$ below the $\mathrm{C} / \mathrm{N}_{0}$ threshold for acquisition.

Figure 1 illustrates three real Delay Doppler Maps (DDMs) obtained using conventional GNSS-R or by the crosscorrelation of the received signal with a clean replica of the transmitted one for different Doppler frequency shifts [10]. The presence of coherent scattering is clearly seen in the shape of the DDMs over sea ice and over land, and they look like the so-called Woodward ambiguity function (WAF) of the transmitted signal, while over the ocean, DDMs spread over many delay (vertical axis) and Doppler (horizontal axis) cells.

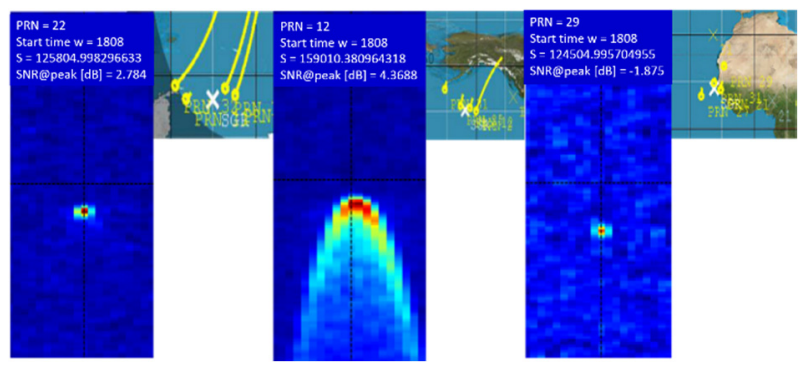

Figure 1. Sample Delay Doppler Maps from the UK TDS-1 mission over ice (left), ocean (center), and land (right). Images from [http://merrbys.co.uk/].

Some reasons explaining this behavior are:

1) for many scattering geometries (i.e. incidence angles), the long electromagnetic wavelength (e.g. $\lambda=19 \mathrm{~cm}$ at $f_{\mathrm{L} 1}=1575.42 \mathrm{MHz}$ ) allows the surface to be considered "smooth," despite natural surfaces exhibit a wide range of root mean square (rms) heights and correlation lengths,

2 ) due to the long chip duration ( $\mathrm{T}_{\text {chip }} \approx 1 \mu \mathrm{s}$ for the GPS $\mathrm{C} / \mathrm{A}$ code, $\mathrm{c} \cdot \mathrm{T}_{\text {chip }} \approx 300 \mathrm{~m}$ ), volumetric effects are usually not resolved, and

3) due to the short coherent integration times $\left(\mathrm{T}_{\mathrm{coh}}=1 \mathrm{~ms}\right)$, in many cases, changes in the phase of contributions from individual points on the Earth's surface may be small within a coherent integration, and at the same time the product of the ground-track speed times the coherent integration time ( $\mathrm{v}_{\text {ground-track }} \cdot \mathrm{T}_{\mathrm{coh}}$ ) is shorter than the correlation length of the surface [11] ( 6-7 $\mathrm{m}$ from space).

Note that the second condition was not satisfied in [4] because of the use of wide bandwidth codes ( $\mathrm{T}_{\text {chip }} \approx 0.1 \mu \mathrm{s}$ ), and that is why different returns were detected (figs. 5 and 11 of [4]). However, due to the slow speed $(\sim 28 \mathrm{~m} / \mathrm{s})$ 
despite the long coherent integration time $\left(\mathrm{T}_{\mathrm{coh}}=20 \mathrm{~ms}\right)$, the third condition was ( $V_{\text {ground-track, balloon }} \cdot T_{\text {coh }} \approx 60 \mathrm{~cm}$ ).

When coherent scattering occurs, the GNSS reflectivity values show very fine details (i.e. very high spatial resolution), as most of the power is mainly coming from the first Fresnel zone. This is illustrated in Fig. 2 (blue dots, reflections over a water channel and colors changing over different fields) with GNSS-R data gathered from $\sim 1 \mathrm{~km}$ height using the LARGO instrument [12].

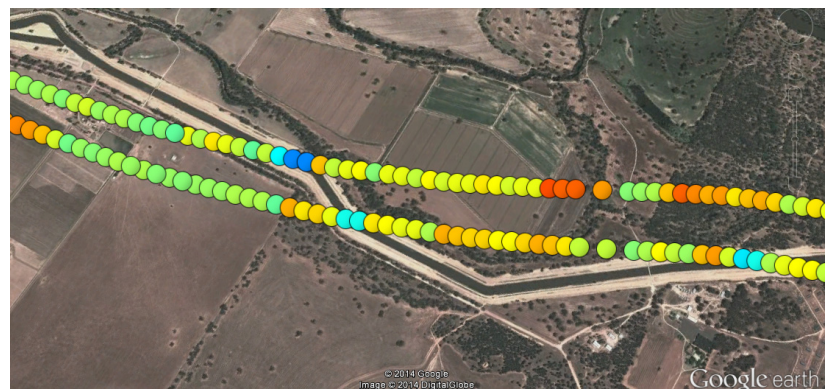

Figure 2. Sample GNSS-R data gathered in Yanco (NSW, Australia) from $1 \mathrm{~km}$ height. Note the reflectivity changes due to terrain inhomogeneity, and the large contrasts when coherent reflections occur over the irrigation water channel.

Under coherent scattering conditions the spatial resolution is usually quantified as an ellipse given by the projection of the first $(n=1)$ Fresnel zone [10]:

$$
\begin{array}{r}
a=R_{n}=\sqrt{n \cdot \lambda \cdot \frac{R_{10} \cdot R_{20}}{R_{10}+R_{20}}}, \\
b=R_{n} / \cos \left(\theta_{i}\right)
\end{array}
$$

where $R_{1}$ is the so-called radius of the first Fresnel zone, $\lambda$ is the electromagnetic wavelength, $R_{10}$ and $R_{20}$ are the distances from the transmitter and receiver to the specular reflection point, and $\theta_{i}$ is the local incidence angle. In the conditions of fig. 2, the pixels' size is $\sim 25 \mathrm{~m}$ at nadir.

Fresnel zones are ellipsoidal shaped regions in the space, with the transmitter and the receiver in their foci. The first region includes the ellipsoidal space where the specular reflection path signal passes through. Its radius $\left(R_{1}\right.$, fig. 3 for a normal incidence) corresponds to a path difference with respect to the line-of-sight of less than half wavelength $(\Delta r \leq \lambda / 2)$, or less than a $\Delta \phi \leq 180^{\circ}$ phase shift. The second region surrounds the first one, but excludes it, and it corresponds to path differences from half-wavelength to one wavelength, i.e. phase shifts $180^{\circ} \leq \Delta \phi \leq 360^{\circ}$. This means that within the first Fresnel zone, all paths with $\Delta r \leq$ $\lambda / 4$ or $\Delta \phi \leq 90^{\circ}$ will contribute positively to the received power, around $r=R_{1}$, when $90^{\circ} \leq \Delta \phi \leq 270^{\circ}$, the received signals will tend to cancel those travelling closer to the lineof-sight, and so on.

For the sake of simplicity, this effect is illustrated in fig. 3 for a nadir specular reflection. The receiver $(R)$ is at a height $h_{R}\left(h_{R} \equiv R_{20}\right)$ over a flat surface, and the specular image of a transmitter ( $\left.\mathrm{T}^{\prime}\right)$ at a height $\mathrm{h}_{\mathrm{T}}\left(\mathrm{h}_{\mathrm{T}} \equiv \mathrm{R}_{10}\right)$ under the surface. The radius of the first Fresnel zone $\left(R_{1}\right)$ is also indicated.

In the above conditions, in the transmission from $\mathrm{T}$, the electric field received at $\mathrm{R}$ from a point in the surface at a distance $\rho$ from the line-of-sight will have approximately the same amplitude as the line-of-sight:

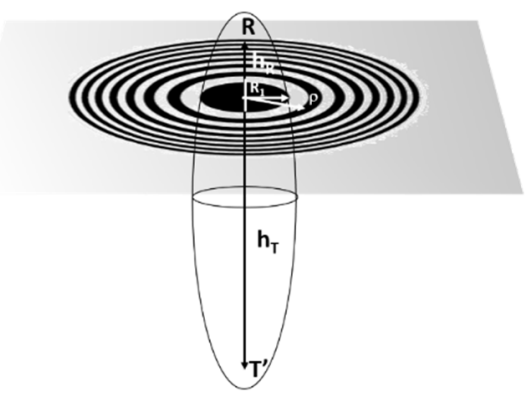

Figure 3. Definition of the coherent scattering geometry for a normal incidence. $\mathrm{R}$ : receiver, $\mathrm{T}$ ': specular image of the transmitter, $R_{1}$ : radius of first Fresnel zone. Black and gray disks represent the odd/even Fresnel zones.

$$
\sqrt{h_{T}^{2}+\rho^{2}}+\sqrt{h_{R}^{2}+\rho^{2}} \approx h_{T}+h_{R},
$$

but to account for the phase difference a second order approximation is needed:

$$
\sqrt{h_{T}^{2}+\rho^{2}}+\sqrt{h_{R}^{2}+\rho^{2}}-h_{T}-h_{R} \approx \frac{\rho^{2}}{2}\left(\frac{1}{h_{T}}+\frac{1}{h_{R}}\right) .
$$

Under these approximations, the power received from a disk of radius $r$ can be computed as the square of the modulus of the total received electric field:

$$
\begin{aligned}
\left|\vec{E}_{t o t, p}(r)\right|= & \frac{E_{T}}{\left(h_{T}+h_{R}\right)} \cdot\left|\int_{0}^{2 \pi} \int_{0}^{r} e^{-j \frac{k}{2}\left(\frac{1}{h_{R}}+\frac{1}{h_{T}}\right) \cdot \rho^{2}} \rho d \rho d \varphi\right| \\
& \propto 2 \cdot\left|\sin \left(\frac{\pi}{2 \lambda} \cdot\left(\frac{1}{h_{R}}+\frac{1}{h_{T}}\right) \cdot r^{2}\right)\right| .
\end{aligned}
$$

This behavior is show in Fig. 4 for a receiver at $h_{R}=500 \mathrm{~km}$, a transmitter at $h_{T}=20000 \mathrm{~km}$, and a wavelength of $\lambda=19 \mathrm{~cm}$ (GPS L1 or Galileo E1). It is important to note the very large fluctuations of the received power, from zero, to a value 4 times larger than in free space, as the received electric field doubles when all subtracting Fresnel zones are masked (i.e. not included in the integral).

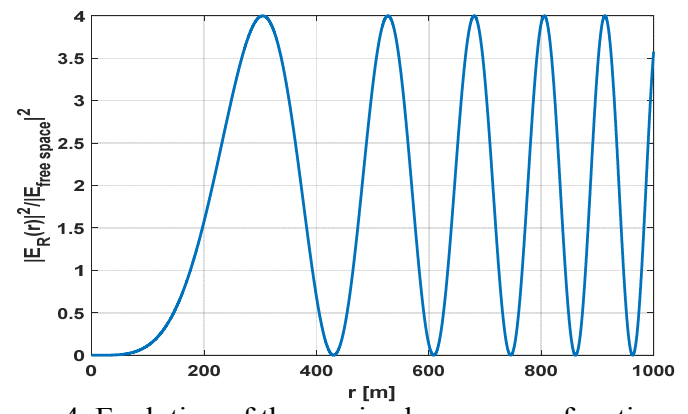

Figure 4. Evolution of the received power as a function of the disk radius " $r$ " normalized to the power received in free space.

Of course, this situation will not happen in a real scenario, but the reflectivity of the targets over the scattering surface will likely introduce a strong modulation of the received power in consecutive observations, e.g. when a small water body producing a strong reflection is seen moving through different 
"Fresnel zones.". This is especially noticeable over land, where the DDM peak shows a very large scattering that is often attributed to speckle noise etc., but actually, for the same coherent and incoherent integration times (typically 1 $\mathrm{ms}$, and $1 \mathrm{~s}$, respectively) the observed scattering is much larger than over homogeneous regions (i.e. sea ice).

In this work, the coherent scattering model of $[13,14]$ is extended to analyze the spatial resolution that can be expected from a GNSS-R instrument when coherent scattering is dominating.

In Section 2, the scattering geometry presented in the introduction will be generalized to include arbitrary incidence angle, and the effect of surface roughness. Finally, numerical results will be presented and discussed in Section 3.

\section{NUMERICAL STUDY FOR ARBITRARY INCIDENCE AND SURFACE ROUGHNESS}

The more general scattering geometry is presented in Fig. 5. For the spherical waves, far away from the first Fresnel zone, the phase changes are fast, and Earth's curvature and wavefront sphericity cannot be neglected. These hypotheses will be validated afterwards, when the size of the scattering region is evaluated and found to be on the order of the Fresnel zone, at most a couple of kilometers even for a typical spaceborne scenario and large incidence angles. When there are rapid variations for the coherent wave, the contribution of the coherent wave becomes negligible and incoherent scattering will dominate.

The specular reflection point is centered in the origin of coordinates $(0,0,0)$, the transmitter $T$ is located at a height $h_{T}$

$$
\overrightarrow{O T}=\left(0,-\tan \left(\theta_{i}\right), 1\right) \cdot h_{T}
$$

and the receiver $R$ is located at a height $h_{R}$

$$
\overrightarrow{O R}=\left(0,+\tan \left(\theta_{i}\right), 1\right) \cdot h_{R}
$$

Root mean square (rms) height $(\sigma)$ is assumed to be small enough so as to preserve the coherence of the forward scattered field. At each point $P$ in the surface, defined by its azimuth angle $\varphi$ and distance $\rho$ to the origin:

$$
\overrightarrow{O P}=(\cos (\varphi), \sin (\varphi), 0) \cdot \rho,
$$

that is contributing to the scattering at the receiver, the scattering coefficient is modelled as [15]:

$$
\sigma^{0} \propto \exp \left(-\left(\beta / \sigma_{s}\right)^{2}\right)
$$

where $\beta(\rho, \varphi)=\operatorname{acos}\left(\widehat{P R}^{\prime} \cdot \widehat{P R}\right)$ is the angle from the direction of specular reflection for that particular location ( $P$, fig. 5):

$$
\overrightarrow{P R^{\prime}}=\left(\rho \cdot \cos (\varphi), \rho \cdot \sin (\varphi)+\tan \left(\theta_{i}\right) \cdot h_{T},+h_{T}\right)
$$

and

$$
\overrightarrow{P R}=\left(-\rho \cdot \cos (\varphi),-\rho \cdot \sin (\varphi)+\tan \left(\theta_{i}\right) \cdot h_{R},+h_{R}\right),(10
$$

and $\sigma_{s}$ is the rms surface roughness slope. For an isotropically rough surface with a Gaussian correlation function, $\sigma_{s}$ is related to the rms height $\sigma$ by the correlation length $(L)$ as:

$$
\sigma_{S}^{2}=\frac{2 \cdot \sigma^{2}}{L^{2}}
$$

and typical values of $\sigma_{s}$ (surface rms slope) range typically from 0.1 to 0.17 over land [15]. This rms height represents that of the surface within approximately the first Fresnel zone. As it will be shown in Section 3, the introduction of the directional behavior in eqn. (8) onwards is required to account for the attenuation of the scattered signals away from the specular direction. Otherwise, as in the flat case (fig. 4), the received power will exhibit large oscillations. The actual value of the surface forward scattering coefficient has to be normalized so that its integral over a hemisphere is equal to an effective reflection coefficient:

$$
\frac{1}{4 \pi \cos \left(\theta_{i}\right)} \iint_{2 \pi}\left(\sigma_{p p}^{0}+\sigma_{p q}^{0}\right) \cdot d \Omega_{s}=\Gamma_{p}
$$

Neglecting cross-polarization effects $\left(\sigma_{p q}^{0}=0\right)$, a mathematically convenient approximation of the forward scattering coefficient at $p$-polarization $\sigma_{p p}^{0}$ is given by:

$$
\sigma_{p p}^{0} \approx 4 \pi \cdot \Gamma_{p} \cdot \frac{\cos \left(\theta_{i}\right)}{\sin \left(\theta_{i}\right)} \frac{2}{\sqrt{\pi} \cdot \sigma_{s}} \cdot \exp \left(-\left(\beta / \sigma_{s}\right)^{2}\right)
$$

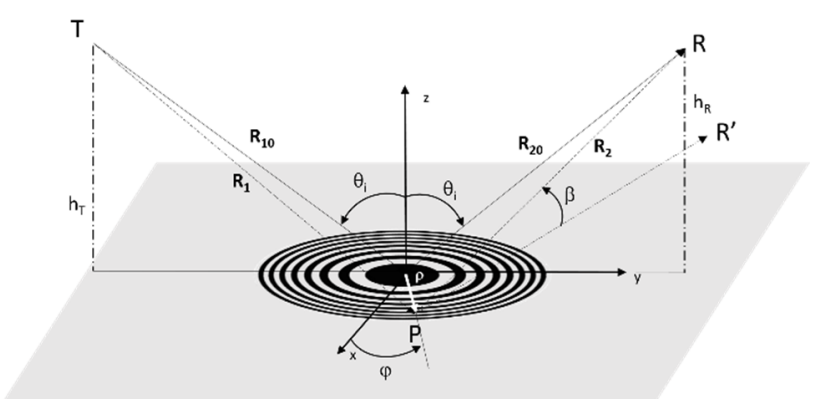

Figure 5. Definition of the coherent scattering geometry. Black and gray disks represent the Fresnel zones that add or subtract to the total electric field depending on the phase difference.

where the power reflection coefficient at $p$-polarization $(\hat{p})$ $\Gamma_{p}=\left|\Gamma_{p, \text { spec }}\right|^{2} \cdot \exp \left(-4 k^{2} \sigma^{2} \cos ^{2}(\theta)\right)$, is equal to the square of the modulus of the complex electric field Fresnel reflection coefficient at that polarization $\left(\Gamma_{p, s p e c}\right)$, which depends on the incidence angle and the soil dielectric constant (i.e. soil moisture...), multiplied by an exponential that accounts for the roughness effects, and $k=2 \cdot \pi / \lambda$. The term $\sigma_{s}$ in the denominator is included to satisfy eqn. (12) when $\sigma_{s} \rightarrow 0$. The amplitude reduction factor "exp $\left(-4 k^{2} \sigma^{2} \cos ^{2}(\theta)\right)$ " significantly attenuates the coherent component for rough surfaces, when $\sigma$ is larger than a few tenths of the wavelength. In the case of sea ice, with a rms height of $3 \mathrm{~cm}$, the attenuation factor is $0.02(\sim$ $-17 \mathrm{~dB})$ at nadir incidence, and $0.14(\sim-8.5 \mathrm{~dB})$ at $45^{\circ}$. 
However, this coherent component is still detectable as demonstrated in [4-7, 9]. It will be shown in what follows that this approximate description of the local scattering cross-section has little impact on the integration results for spaceborne geometries.

Under the above assumptions, the coherent component of the electric field scattered from point $\mathrm{P}$ into the direction of the receiver is given by:

$$
\begin{gathered}
d \vec{E}_{t o t, p}(\rho, \varphi)=\frac{E_{T}}{\sqrt{4 \pi} \cdot\left(R_{1}+R_{2}\right)} \cdot \sqrt{4 \pi} \cdot \Gamma_{p, s p e c} \cdot e^{-2 k^{2} \sigma^{2} \cos ^{2}(\theta)} . \\
\sqrt{\frac{\cos \left(\theta_{i}\right)}{\sin \left(\theta_{i}\right)} \frac{2}{\sqrt{\pi} \cdot \sigma_{S}}} \cdot e^{-j k\left(R_{1}+R_{2}\right)} \cdot e^{-\frac{1}{2}\left(\frac{\beta}{\sigma_{S}}\right)^{2}} \cdot \hat{p}
\end{gathered}
$$

As in Section 1, but with a slightly more complicated Math, and making use of:

$$
\begin{aligned}
& R_{1}=\sqrt{\rho^{2} \cdot \cos ^{2}(\varphi)+\left(h_{T} \cdot \tan \left(\theta_{i}\right)+\rho \cdot \sin (\varphi)\right)^{2}+h_{T}^{2}},(15 \mathrm{a}) \\
& R_{2}=\sqrt{\rho^{2} \cdot \cos ^{2}(\varphi)+\left(h_{R} \cdot \tan \left(\theta_{i}\right)-\rho \cdot \sin (\varphi)\right)^{2}+h_{R}^{2}},(15 \mathrm{~b})
\end{aligned}
$$

the following approximations apply for the phase:

$$
\begin{aligned}
R_{1}+R_{2} \approx R_{10}+R_{20}+\left(\frac{1}{h_{R}}+\frac{1}{h_{T}}\right) \cdot \rho^{2} \cdot \frac{\cos \left(\theta_{i}\right)}{2} . \\
\left(1-\sin ^{2}\left(\theta_{i}\right) \cdot \sin ^{2}(\varphi)\right)
\end{aligned}
$$

and for the amplitude:

$$
R_{1}+R_{2} \approx R_{10}+R_{20}
$$

where $R_{i 0}=R_{i}(\rho=0), i=1,2$, and then eqn. (14) becomes:

$d \vec{E}_{t o t, p}(\rho, \varphi)=\frac{E_{T}}{\left(R_{10}+R_{20}\right)} \cdot \Gamma_{p, s p e c} \cdot e^{-2 k^{2} \sigma^{2} \cos ^{2}(\theta)} \cdot \sqrt{\frac{\cos \left(\theta_{i}\right)}{\sin \left(\theta_{i}\right)} \frac{2}{\sqrt{\pi} \cdot \sigma_{s}}} \cdot$ $e^{-\frac{1}{2}\left(\frac{\beta}{\sigma_{S}}\right)^{2}} \cdot e^{-j k\left(R_{10}+R_{20}\right)} e^{-j k\left(\frac{1}{h_{R}}+\frac{1}{h_{T}}\right) \cdot \rho^{2} \cdot \frac{\cos \left(\theta_{i}\right)}{2} \cdot\left(1-\sin ^{2}\left(\theta_{i}\right) \cdot \sin ^{2}(\varphi)\right)} \hat{p}$.

Finally, since the area contributing to coherent scattering is small, the variations of the local incidence angle and the rotation of the polarization reference frame are negligible. The amplitude of the total electric field received at the receiver $R$ from all points inside a circle of radius $r$ centered in the specular reflection point $(0,0,0)$ can be computed as the coherent addition of all the contributions in the area:

$$
\begin{aligned}
& \left|\vec{E}_{t o t, p}(r)\right|=\frac{E_{T}}{\left(R_{10}+R_{20}\right)} \cdot\left|\Gamma_{p, s p e c}\right| \cdot e^{-2 k^{2} \sigma^{2} \cos ^{2}(\theta)} \cdot \sqrt{\frac{\cos \left(\theta_{i}\right)}{\sin \left(\theta_{i}\right)} \frac{2}{\sqrt{\pi} \cdot \sigma_{S}}} \cdot \\
& \left|\int_{0}^{2 \pi} \int_{0}^{r} e^{-j k\left(\frac{1}{h_{R}}+\frac{1}{h_{T}}\right) \cdot \rho^{2} \cdot \frac{\cos \left(\theta_{i}\right)}{2} \cdot\left(1-\sin ^{2}\left(\theta_{i}\right) \cdot \sin ^{2}(\varphi)\right)} e^{-\frac{1}{2}\left(\frac{\beta}{\sigma_{S}}\right)^{2}} \rho d \rho d \varphi\right| .
\end{aligned}
$$

\section{RESULTS AND DISCUSSION ON THE SPATIAL RESOLUTION}

As an example, fig. 6 shows the numerical results of the evaluation of eqn. (19) squared and normalized, so that they are independent from the reflection coefficient, and the "normalized Pointing vector" asymptotically tends to 1 when $r \rightarrow \infty$, i.e. all Fresnel zones are accounted for, and the free-space Pointing vector at the receiver becomes:

$$
\lim _{r \rightarrow \infty}\left|\vec{P}_{i}(r)\right|=\frac{\left|E_{T}\right|^{2}}{4 \pi \cdot \eta \cdot\left(R_{10}+R_{20}\right)^{2}},
$$

where $\eta$ is the wave impedance $(120 \pi$ ohms in the vacuum). The parameters used in fig. 6 are: the incidence angle $\theta_{i}$ is set to $1^{\circ}, 15^{\circ}, 30^{\circ}, 45^{\circ}, 60^{\circ}$, and $75^{\circ}, h_{R}=500$ $\mathrm{km}, h_{T}=20000 \mathrm{~km}, \lambda=19 \mathrm{~cm}$, and $\sigma_{S}=0.1$, and it has been computed in steps of $1 \mathrm{~m}$. It must be noted that, while typical values of $\sigma_{s}$ over land are in the range 0.1-0.17 [15], differences in the numerical results are negligible for $\sigma_{s}$ in the range $0.01-1$, and even larger.

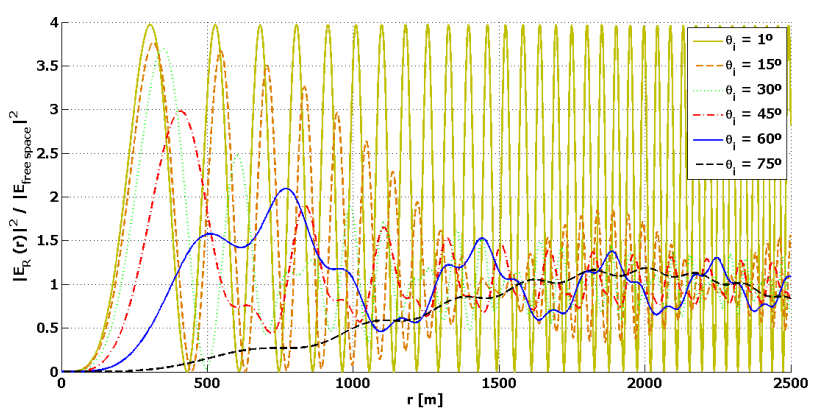

Figure 6. Evolution of the received power as a function of the disk radius " $r$ " normalized to the power received in free space. normalized to the free-space and to the reflection coefficient for different incidence angles.

As it can be appreciated, for near-nadir incidence $\left(\theta_{i}=1^{\circ}\right)$, the received power exhibits peaks (double electric field, four-fold received power) and valleys (received power vanishes), as different Fresnel zones are included in the integration as $r$ increases, as in fig. 4, as for near nadir incidence $\left(\theta_{i} \rightarrow 0^{\circ}\right)$ the Fresnel zones are nearly circular ( $b \rightarrow a$ in eqn. (1)). Then, as the incidence angle increases the Fresnel zones become ellipses that are more and more elongated, the peak power $\left(P_{\max }\right.$, Table $1,2^{\text {nd }}$ column $)$ decreases, and the distance $r$ at which the maximum power is received occurs for larger integration areas (larger $r$ 's or $\left.R\right|_{P_{\text {max }}}$, Table $1,3^{\text {rd }}$ column).

The first value for which the normalized Pointing vector is equal to one $\left(R_{0, e q}\right.$, Table $1,4^{\text {th }}$ column $)$ can be associated to half the spatial resolution (radius of an equivalent circular footprint), since the received power equals that in free-space conditions (apart from the intensity reduction due to the reflection itself). The $5^{\text {th }}$ column in Table 1 shows the ratio between $R_{0, e q}$ and the geometric mean of the semi-major axis $(a)$ and semi-minor axis $(b)$ of the ellipse of the projected first Fresnel zone (radius of a circle with the same area). As it can be observed, this ratio is nearly constant, around $0.58-0.62$, up to $60^{\circ}$ incidence angle. These values are in agreement with the $0.56 \approx$ $2 / \sqrt{\pi}$ value quoted in [16] using a much simpler approach, and can be interpreted as the signal is basically coming from an area smaller than the first Fresnel zone. However, the peak power (up to 4 times more, i.e. $+6 \mathrm{~dB}$, electric field amplitude being twice larger) is received from 
an area that extends up to the $1^{\text {st }}$ Fresnel zone itself $\left(\left.R\right|_{P_{\max }} / \sqrt{a b} \approx 1\right.$, Table 1 , last column). The large intensity oscillations beyond the first Fresnel zone indicate that significant amounts of power can also be collected from regions farther away from the specular region as determined by the first Fresnel zone (up to 1000-1500 m or more, depending on the incidence angle). Over inhomogeneous targets (e.g. land), variations of the reflection coefficient will modulate the received power pattern contributing to the large power scattered observed, that difficult the data interpretation and geophysical parameter retrieval, such as soil moisture or vegetation biomass. These fluctuations can be reduced by spatial averaging, at the expense of a reduction of the spatial resolution, which in any case still is much smaller than in the incoherent case (fig. 1, center).

Table 1. Normalized maximum power $\left(P_{\max }\right)$, radius at which this occurs $\left(\left.R\right|_{P_{\max }}\right)$, and ratio with respect to the effective radius of the first Fresnel zone $\left(\left.R\right|_{P_{\max }} / \sqrt{a b}\right)$. Effective half spatial resolution $\left(R_{0, e q}\right)$, and ratio with respect to the effective radius of the first Fresnel zone $\left(R_{0, e q} / \sqrt{a b}\right)$.

\begin{tabular}{|r|c|c|c|c|c|}
\hline$\theta_{i}$ & $P_{\max }$ & $\left.R\right|_{P_{\max }}[\mathrm{m}$ & $R_{0, e q}$ & $R_{0, e q} / \sqrt{a b}$ & $\left.R\right|_{P_{\max }} / \sqrt{a b}$ \\
\hline $1^{\circ}$ & 3.97 & $304 \mathrm{~m}$ & $176 \mathrm{~m}$ & 0.58 & 1.00 \\
\hline $15^{\circ}$ & 3.76 & $315 \mathrm{~m}$ & $185 \mathrm{~m}$ & 0.59 & 1.00 \\
\hline $30^{\circ}$ & 3.70 & $348 \mathrm{~m}$ & $205 \mathrm{~m}$ & 0.58 & 0.99 \\
\hline $45^{\circ}$ & 2.98 & $408 \mathrm{~m}$ & $253 \mathrm{~m}$ & 0.59 & 0.95 \\
\hline $60^{\circ}$ & 2.10 & $769 \mathrm{~m}$ & $378 \mathrm{~m}$ & 0.62 & 1.26 \\
\hline $75^{\circ}$ & 1.19 & $2000 \mathrm{~m}$ & $1567 \mathrm{~m}$ & 1.33 & 1.70 \\
\hline
\end{tabular}

\section{CONCLUSIONS}

This study has analyzed the spatial resolution in GNSS-R systems when coherent scattering is dominant (i.e. over sea ice and in some conditions land). Results confirm that up to $\sim 45^{\circ}$, most of the power can be interpreted as coming from an area determined by the first Fresnel zone (last column in Table 1), but that a power equivalent to the one that will be received in free-space comes actually from a smaller region ( $5^{\text {th }}$ column of Table 1 ), about $0.58-0.62$ the size of the $1^{\text {st }}$ Fresnel zone. However, since the spatial resolution is not limited by the antenna footprint, non-negligible contributions from regions farther away than the $1^{\text {st }}$ Fresnel zone can be collected, and therefore, large intensity fluctuations can be expected over inhomogeneous targets depending on the scattering coefficient of these regions.

\section{REFERENCES}

[1] S. Gleason et al., "Detection and processing of bistatically reflected GPS signals from low Earth orbit for the purpose of ocean remote sensing", IEEE Trans on Geosci. and Rem. Sens., Vol. 43(6), pp. 1229-1241, 2005. [2] A. Alonso-Arroyo, et al., "Sea Ice Detection Using U.K. TDS-1 GNSS-R Data," IEEE Trans on Geosci. and Rem. Sens., Vol. 55(9), pp. 4989-5001, Sept. 2017.

[3] Li, W., et al., (2017), "First spaceborne phase altimetry over sea ice using TechDemoSat-1 GNSS-R signals," Geophys. Res. Lett., 44, 8369-8376.
[4] H. Carreno-Luengo, et al., "First Results of a GNSS-R Experiment From a Stratospheric Balloon Over Boreal Forests," IEEE Trans on Geosci. and Rem. Sens., Vol. 54(5), pp. 2652-2663, May 2016.

[5] H. Carreno-Luengo and A. Camps, "First Dual-Band Multiconstellation GNSS-R Scatterometry Experiment Over Boreal Forests From a Stratospheric Balloon," in IEEE Journal of Selected Topics in Applied Earth Obs. and Rem. Sens., Vol 9(10), pp. 4743-4751, Oct. 2016.

[6] A. Camps, et al. "Ionospheric Effects in GNSSReflectometry From Space," in IEEE Journal of Selected Topics in Applied Earth Observations and Remote Sensing, Vol. 9(12), pp. 5851-5861, Dec. 2016.

[7] E. Valencia, et al., "Experimental Determination of the Sea Correlation Time Using GNSS-R Coherent Data," in IEEE Geosci. and Rem. Sens. Letters, Vol. 7(4), pp. 675679, Oct. 2010.

[8] C. Chew, et al., "CYGNSS data map flood inundation during the 2017 Atlantic hurricane season," Scientific Reports (2018) 8:9336, DOI:10.1038/s41598-018-27673-x [9] F. Martin et al., "Mitigation of Direct Signal Cross-Talk and Study of the Coherent Component in GNSS-R," in IEEE Geosci. and Rem. Sens. Letters, Vol. 12(2), pp. 279283, Feb. 2015.

[10] V. Zavorotny, et al., "Tutorial on Remote Sensing Using GNSS Bistatic Radar of Opportunity", IEEE Geoscience and Rem. Sens. Magazine, Vol. 2(5), pp. 8-45, 2014.

[11] G.A. Hajj, and C. Zuffada, "Theoretical description of a bistatic system for ocean altimetry using the GPS signal," Radio Science, Volume 38, Issue 5, CiteID 1089, DOI 10.1029/2002RS002787

[12] A. Alonso-Arroyo, et al. "The light airborne reflectometer for GNSS-R observations (LARGO) instrument: Initial results from airborne and Rover field campaigns," 2014 IEEE Geosci. and Rem. Sens. Symposium, Quebec City, QC, 2014, pp. 4054-4057.

[13] A.K. Fung, and H.J. Eom, "Coherent scattering of a spherical wave from an irregular surface", IEEE Trans on Antennas and Prop., Vol. 31(1), pp. 68-72, 1983.

[14] R. D. De Roo, and F.T. Ulaby, "Bistatic Specular Scattering from Rough Dielectric Surfaces," IEEE Trans. Antennas Prop., Vol. 42(2), pp. 220-231, 1994

[15] M.I. Skolnik, "Radar Handbook," 2nd edition, ed. McGraw-Hill, 1990 (chapter 25)

[16] H. Carreno-Luengo and A. Camps, "Unified GNSS-R formulation including coherent and incoherent scattering components," 2016 IEEE Int. Geosci. and Rem. Sens. Symp. (IGARSS), Beijing, 2016, pp. 4815-4818.

ACKNOWLEDGEMETNS: This study has been supported by projects SPOT (RTI2018-099008-B-C21) of the Spanish Ministry of Science, Innovation and Universities, and EU FEDER, ICREA Academia Award from the Catalan Government, and ESA/ESTEC CONTRACT n. $4000120299 / 17 / \mathrm{NL} / \mathrm{AF} / \mathrm{hh}$. The author is also thankful to Drs. Carlos López-Martínez and Hugo Carreno-Luengo for the helpful discussions regarding this topic. 